\title{
The spectral variations of MWC 314
}

\author{
Corinne Rossi $^{1}$, Antonio Frasca ${ }^{2}$, Ettore Marilli $^{2}$, Michael Friedjung ${ }^{3}$, \\ Gérard Muratorio ${ }^{4}$ \\ ${ }^{1}$ Dipartimento di Fisica, Universita La Sapienza, Roma, Italy; \\ email:corinne.rossi@uniroma1.it \\ ${ }^{2}$ INAF - Osservatorio Astrofisico di Catania, Italy; \\ ${ }^{3}$ Institut d'Astrophysique CNRS+Paris 6, Paris, France; ${ }^{4}$ OMP/LAM, Marseille, France
}

\begin{abstract}
New spectra of MWC314 are presented; they indicate that the V/R emission line flux ratios show signs of varying in an opposite way to the absorption line radial velocities. The latter appear to be due to apparently non-periodic pulsations, perhaps in strange modes.
\end{abstract}

Keywords. stars: emission-line, Be, stars: individual (MWC 314)

\section{Introduction}

There has been confusion about whether MWC 314 is a B[e] supergiant or a luminous blue variable. However, it does not show the typical time variations of the latter class. In addition, it appears to be one of the most luminous stars in the Galaxy (Miroshnichenko et al. 1998). Permitted emission line profiles show two peaks, suggesting formation in a rotating disk and we considered it to belong rather to the $\mathrm{B}[\mathrm{e}]$ class (Muratorio et al. 2008). Rapid variations in the displacements of the absorption lines, previouly suggested by Wisniewski et al. (2006), could indicate binarity (Muratorio et al. 2008) with a period of $\sim 31$ days. In order to confirm the suggested periodicity, we obtained additional spectra at the Catania Observatory (OAC) during 2008 and 2009 in the range 4300-6850 $\AA$ with resolution $R \simeq 21000$. In some cases we could follow the star for several consecutive nights.

\section{Data analysis}

The new observations confirmed that: a) The heliocentric radial velocity of the absorption lines is strongly variable, while that of the barycentre of the emission lines remains constant (see Figure 1, from top to bottom: June 15, 20, 24, July 3, 30, August 31, September 4, 2009); b) The intensities of the emission line peaks and the position of the central dip vary (see Figure 2); c) The ratios V/R, obtained from double Gaussian fits of the emission line fluxes, vary in an opposite way to the mean of the absorption line radial velocities (see Figure 3 top and bottom, respectively). This effect does not appear to be explicable by blending of the absorption lines and the emission line peaks, suggesting a more profound physical effect. There is no direct connection between the absorption lines and the minimum between the two peaks of each emission line. In any case more analysis must be undertaken. The periodicity of the radial velocity variation is not confirmed. Though binarity is still possible, all the radial velocity variations cannot be so explained. Strange modes of pulsation (see the review by Glatzel \& Chernigovski, 2001) which can be chaotic, have been invoked to explain the variations of luminous blue variables and especially massive stars for which the luminosity/mass ratio in solar units is more than $10^{3}$. That appears to be the case for our star. 

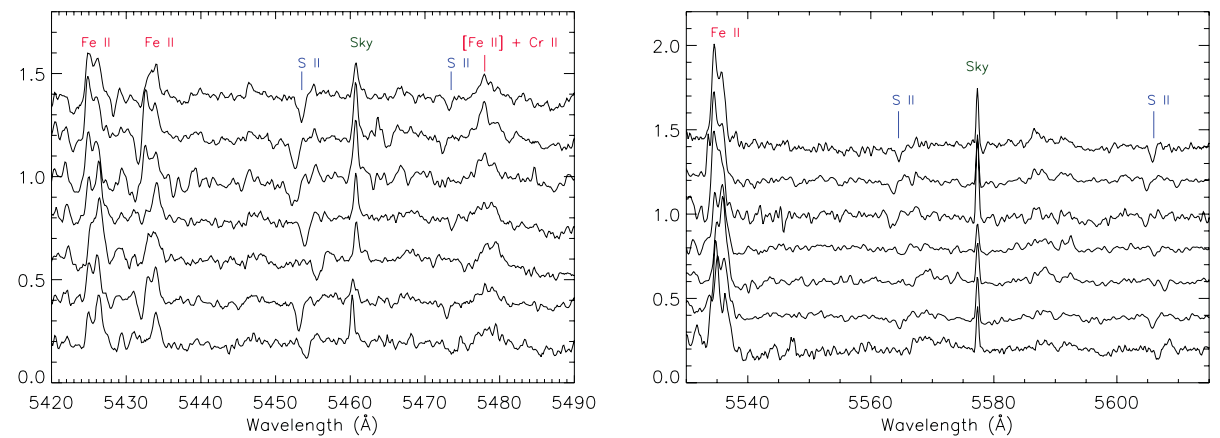

Figure 1. Selected regions from spectra taken at OAC in Summer 2009.
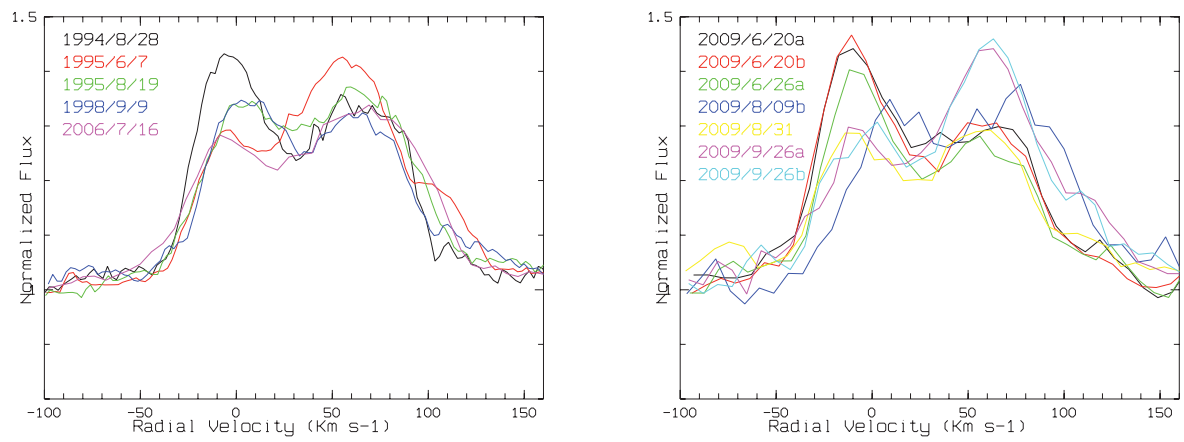

Figure 2. Fe II line $6416.90 \AA$ A: Older observations, left. Recent data from OAC, right.
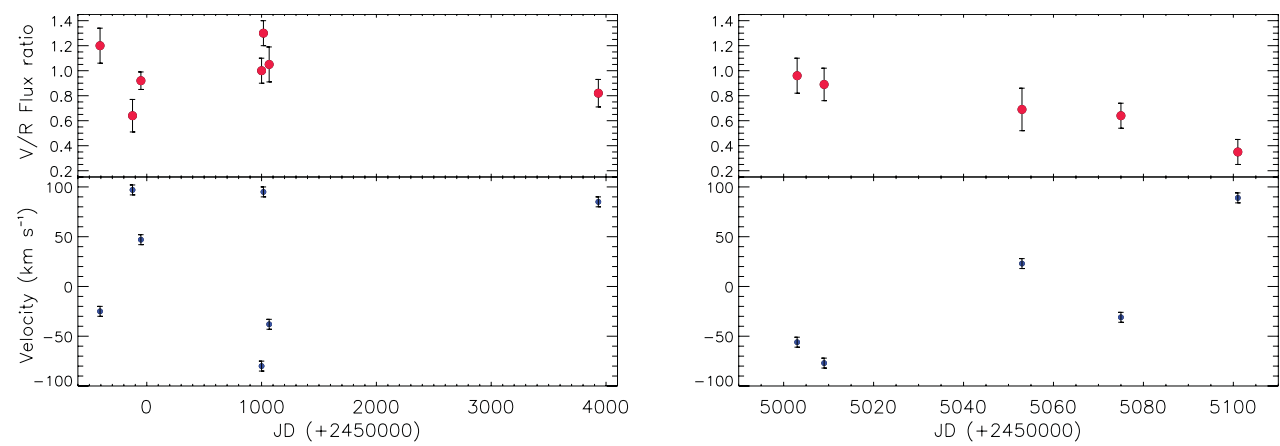

Figure 3. V/R ratios of permitted emissions (top panels) and radial velocities of the absorptions as a function of JD (lower panels). Older observations, left. Recent data from OAC, right.

\section{References}

Miroshnichenko, A. S., Fremat, Y., Houziaux, L., Andrillat, Y. et al. 1998, A\&SAS, 131, 469

Glatzel, W. \& Chernigovski, S. 2001, in: M. de Groot \& C. Sterken (eds.), P Cygni 2000: 400 Years of Progress, ASP-CS, 233, p. 227

Muratorio, G., Rossi, C. \& Friedjung, M. 2008, A\&A 487, 637

Wisniewski, J. P., Babler, B. L., Bjorkman, K. S., Kurchakov, A. V. et al. 2006, PASP, 118, 820 